\title{
Dysregulation of zinc finger protein, $X$-linked (ZFX) impairs cell proliferation and induces apoptosis in human oral squamous cell carcinorma
}

\author{
Hongzhi Ma • Fan Yang • Meng Lian • Ru Wang • \\ Haizhou Wang $\cdot$ Ling Feng $•$ Qian Shi $\cdot$ Jugao Fang
}

Received: 1 September 2014 / Accepted: 26 February 2015 / Published online: 28 April 2015

(C) The Author(s) 2015. This article is published with open access at Springerlink.com

\begin{abstract}
Zinc finger protein, X-linked (ZFX) is a transcriptional factor involved in many physiological processes such as embryonic stem cell survival and self-renewal. Though ZFX dysfunctions have been identified in variant human diseases and especially in cancers, its pathological roles have not been fully addressed. Here, we explored the relationship between ZFX expression and squamous cell carcinoma (SCC) of the tongue. We found that ZFX expression was significantly higher in tongue SCC tumors as compared to tumor-adjacent normal tissues. Furthermore, ZFX knockdown impeded cell proliferation, impaired colony formation ability, and lead to cell cycle arrest while induced cell apoptosis in human tongue squamous cell carcinoma cell line Tca-8113. Our results provide evidence suggesting that ZFX overexpression is associated with the development of tongue SCC and ZFX knockdown is a potential treatment for tumor suppression.
\end{abstract}

Keywords Oral squamous cell carcinorma $\cdot$ Zinc finger protein, X-linked $\cdot$ Proliferation $\cdot$ Cell cycle $\cdot$ Apoptosis

H. Ma $\cdot$ F. Yang $\cdot$ M. Lian $\cdot$ R. Wang $\cdot$ H. Wang $\cdot$ L. Feng $\cdot$ Q. Shi $•$ J. Fang $(\square)$

Department of Otolaryngology-Head and Neck Surgery, Beijing

Tongren Hospital, Capital Medical University, Beijing 100730,

China

e-mail: fangjugao@vip.sohu.com

H. Ma $\cdot$ J. Fang

Key Laboratory of Otorhinolaryngology Head and Neck Surgery,

Ministry of Education, Beijing Institute of Otorhinolaryngology,

Beijing 100005, China

\section{Introduction}

Tongue squamous cell carcinoma (SCC) is the most common malignant type in head and neck squamous cell carcinoma (HNSCC), which is among the top ten leading cancers worldwide [1]. Though remarkable progress has been achieved in clinical and basic research in the past three decades, the 5-year survival rate of tongue SCC patients showed little improvement and remained about $50 \%$ [2]. Another major problem is that most tongue SCC cases showed lymph node metastasis at diagnosis, which indicated poor prognosis, and effective treatment are still underway at present. Though multiple factors including drinking and smoking have been implicated in the development and progression of tongue SCC [3], molecular signals and factors involved in tumorigenesis remain to be determined. Exploring novel molecules involved in tongue SCC will provide valuable insights for better diagnosis and treatment of human tongue $\mathrm{SCC}$ in future clinical studies.

$\mathrm{Cys}_{2} \mathrm{His}_{2}$ zinc finger $(\mathrm{C} 2 \mathrm{H} 2-\mathrm{ZF})$ proteins are the major class of DNA-binding proteins and have been implicated in diverse biological processes such as cell proliferation differentiation and cell survival while their dysfunctions have been linked to multiple human diseases including variant types of cancer [4]. Zinc finger protein, X-linked (ZFX) which is encoded by $\mathrm{X}$-linked ZFX gene is a $\mathrm{C} 2 \mathrm{H} 2-\mathrm{ZF}$ transcriptional factor composed of three functional domains including a DNA-binding domain containing $13 \mathrm{C} 2 \mathrm{H} 2$-type zinc fingers, an acidic transcriptional activation domain, and a nuclear localization sequence. It has been shown previously that ZFX is critical for the survival and self-renewal of embryonic and hematopoietic stem cells $[5,6]$. In consistent with the role of $\mathrm{C} 2 \mathrm{H} 2-\mathrm{ZF}$ proteins in cancer development, previous studies from our lab have shown that ZFX is aberrantly upregulated in human laryngeal squamous cell carcinoma and ZFX knockdown impaired cancer cell proliferation and apoptosis [7]. 
Furthermore, ZFX protein has been implicated in acute myeloid leukemia (AML) and acute T-lymphoblastic leukemia (TALL) [8]. However, the relationship between ZFX and tongue SCC remains poorly understood.

As both tongue SCC and laryngeal squamous cell carcinoma belong to HNSCC, here we examined the expression status of ZFX and unraveled significant expression differences between tumors and adjacent normal tissues derived from tongue SCC patients. In accord with previous studies showing that ZFX expression is increased in tumors, ZFX overexpression was observed in tongue SCC samples. Furthermore, ZFX expression in human tongue squamous cell carcinoma cell line Tca-8113 was inhibited efficiently by lentiviral-based small interference RNA (siRNA) strategy, and the impact of ZFX knockdown on cell proliferation, colony formation, cell cycle, and cell survival was investigated extensively, confirming the pathological role of ZFX for tongue SCC development and progression. So, our results provide confidential evidence that ZFX is quite a promising target for tongue SCC treatment.

\section{Materials and methods}

Patients, tumor samples, and immunohistochemistry (IHC) staining

This study was approved by the Research Ethics Board, and informed consent was obtained from all patients. A consecutive series of 30 tongue squamous cell carcinoma patients including 21 male and 9 female patients (between 24 and 85 years old with an average 59.7 years old) who underwent surgery between March 2011 and December 2013 was involved in this study. Patients could be classified according to tumor TNM stage system and shown in Table 1. None of the patients were treated with radiotherapy, chemotherapy, or other therapies before surgery. Specimens of tumor and of normal adjacent tissues were obtained, frozen in liquid nitrogen immediately, and stored at $-80{ }^{\circ} \mathrm{C}$ for further analysis. ZFX immunohistochemical staining was performed as described previously [9]. Briefly, 4.0- $\mu \mathrm{m}$-thick paraffin-embedded
Table 1 Patient information

\begin{tabular}{lc}
\hline $\begin{array}{l}\text { Clinical pathological } \\
\text { parameters }\end{array}$ & $\begin{array}{l}\text { Case } \\
\text { number }\end{array}$ \\
\hline Gender & 21 \\
Male & 9 \\
Female & \\
TNM stage & 4 \\
T1N0M0 & 18 \\
T2N0M0 & 1 \\
T2N2M0 & 7 \\
T3N0M0 & \\
\hline
\end{tabular}

tissue sections were prepared and incubated with NBP180582 ZFX antibody purchased from Novus Company. Staining was done with an EliVision ${ }^{\mathrm{TM}}$ plus kit (Fuzhou Maixin Biotech. Co., Ltd). Then, in each slice, five fields were selected randomly under high magnification $(400 \times)$. The mean optical density (OD) of ZFX positive cells was counted in each field, and then, OD value of five fields was averaged for ZFX expression in each slice. OD value of each specimen was calculated as above, and paired $t$ test was used to for OD value comparation between tumor and normal adjacent tissue samples. Leica dc300f microscope, Leica IM50 picture collecting system, and Leica Qwin software were used here.

\section{Production of lentivirus expressing ZFX-siRNA}

To inhibit ZFX expression, lentivirus expressing siRNA targeting ZFX specifically was produced as described previously [10]. Briefly, siRNA (siRNA sequence: gaGCCTGA GAATGATCATGGA) specifically targeting human ZFX gene (ZFX-siRNA) was designed while scrambled siRNA (scr-siRNA) with the following sequence was used as the negative control: TTCTCCGAACGTGTCACGT. To construct lentivirus expressing ZFX- or scr-siRNA, complementary single-strand DNA oligonucleotides were synthesized, annealed, and then inserted into lentiviral vector pGCSILGFP (GeneChem, Shanghai, China). Lentivector expression system (GeneChem, Shanghai, China) was selected to prepare desired lentivirus. Human tongue squamous cell carcinoma cell line Tca-8113 was selected and infected with lentivirus expressing ZFX- or scr-siRNA to determine the knockdown efficiency at RNA and protein level by real-time PCR and Western blot.

\section{Bromodeoxyuridine (BrdU) incorporation assay}

Cell proliferation was assessed using BrdU incorporation assay. Human tongue squamous cell carcinoma cell line Tca8113 was cultured and infected with lentivirus expressing ZFX or scrambled siRNA and incubated for another $48 \mathrm{~h}$. Cells were then resuspended and seeded into 96-well plates at the proper density and cultured for another 1 or 4 days. Then, BrdU reagents were added with 1:100 dilution $(10 \mu \mathrm{l} /$ well). After 2-24 h, BrdU incorporation was analyzed using a BrdU Cell Proliferation ELISA kit (Cat. No. 11647229001, Roche Applied Science) following the manufacturer's instructions. Briefly, cell fixation was performed using FixDenat (200 $\mu \mathrm{l} /$ well) for $30 \mathrm{~min}$ and blocked with $5-10 \%$ BSA for another $30 \mathrm{~min}$ at room temperature. Then, cells were treated with diluted anti-BrdU-POD for $90 \mathrm{~min}$ at room temperature. Cells were incubated with substrate solution for 5-30 $\mathrm{min}$ in the dark after washing three times with washing buffer. After color development, the absorbance of the samples was measured at $450 \mathrm{~nm}$ to determine BrdU incorporation ratio. 


\section{MTT assay}

Cell proliferation status was determined by MTT assay. Human tongue squamous cell carcinoma cell line Tca-8113 was infected with lentivirus expressing ZFX-siRNA or scrambled siRNA and incubated to reach logarithmic phase. Cells were then digested and resuspended thoroughly. Cell number was then counted using a hemocytometer, and 3000 cells per well were added in 96 -well plates and cultured at $37^{\circ} \mathrm{C}$ in a $5 \%$ $\mathrm{CO}_{2}$ incubator. Cell proliferation was monitored for continuous 5 days with MTT assay. Each well was incubated with $20 \mu \mathrm{l}$ of MTT solution $(5 \mathrm{mg} / \mathrm{ml})$ for $4 \mathrm{~h}$. The culture medium was discarded, and $150 \mu \mathrm{l}$ DMSO was added to dissolve formazan. 96-well plate was shaken for 5-10 $\mathrm{min}$, and absorbance at 490/570 $\mathrm{nm}$ was examined with a microplate reader.

\section{Cell apoptosis measurement}

Cell apoptosis was detected using annexin V-APC apoptosis detection kit (eBioscience, 88-8007) and measured with flow cytometry. In brief, Tca- 8113 cells were infected with lentivirus expressing ZFX-siRNA or scrambled siRNA and incubated at $37{ }^{\circ} \mathrm{C}$ for 4 days. After collection and washing with phosphate-buffered saline (PBS) buffer, cells were resuspended with staining buffer at a final density of $1 \times 10^{6}-1 \times 10^{7} / \mathrm{ml}$. Then, $5 \mu \mathrm{l}$ annexin V-APC was added to $100 \mu \mathrm{l}$ cell suspensions and incubated at room temperature in the dark for 1015 min. Finally, cells were analyzed with FACS Calibur (Becton-Dickinson, USA) to determine cell apoptosis profiles.

RNA extraction, reverse-transcription, and real-time quantitative PCR

To determine the siRNA efficiency, Tca- 8113 cells were infected with lentivirus expressing either ZFX-siRNA or scrambled siRNA and cultured for another $48 \mathrm{~h}$. Then, total RNA was isolated using Trizol reagent (Invitrogen) according to the manufacturer's instructions. M-MLV reverse transcriptase (Promega) and Oligo dT primers (Sangon, Shanghai) were used for reverse transcription to produce cDNAs. Briefly, a $10 \mu \mathrm{l}$ mixture containing $2 \mu \mathrm{g}$ RNA, $0.5 \mu \mathrm{g}$ Oligo dT primers was incubated at $70{ }^{\circ} \mathrm{C}$ for $10 \mathrm{~min}$ and then cooled on ice. Then, buffer, reverse transcriptase, RNase inhibitor, and dNTPs were added to a final $20 \mu \mathrm{l}$ mixture and incubated at $42{ }^{\circ} \mathrm{C}$ for $1 \mathrm{~h}$. ZFX expression was quantified with real-time quantitative PCR using a Real-Time PCR machine TP800 (Takara). Here, glyceraldehyde-3-phosphate dehydrogenase $(\mathrm{GAPDH})$ was used as an internal control. Primers used in real-time PCR were as follows: GAPDH for 5'-TGACTTCA ACAGCGACACCCA-3'; GAPDH reverse: 5'-CACCCTGT TGCTGTAGCCAAA-3'; ZFX for 5'-GGCAGTCCACAG CAAGAAC-3'; ZFX reverse: 5'-TTGGTATCCGAGAAAG TCAGAAG-3'. Messenger RNA (mRNA) levels of ZFX were normalized against GAPDH, and the comparative $\mathrm{C}_{\mathrm{T}}$ method was chosen to quantify ZFX expression in Microsoft Excel.

\section{Colony formation assay}

In brief, lentivirus expressing either ZFX-siRNA or scrambled siRNA was added into Tca-8113 cells. After incubation for another $48 \mathrm{~h}$, cells reached the logarithmic phase and were harvested. Then, cell density was calculated using a hemocytometer, and 800 cells per well were plated in triplicate into 6well plates. After culturing in a $5 \% \mathrm{CO}_{2}$ incubator at $37^{\circ} \mathrm{C}$ for another 14 days, cells were fixed with paraformaldehyde for 30-60 min and then stained with GIEMSA for $20 \mathrm{~min}$. Micropublisher 3.3RTV (Olympus) was used for imaging capture, and then, cell colonies under each condition were counted and analyzed.

\section{Cell cycle analysis by flow cytometry}

Cell cycle process was analyzed using flow cytometry as described in previous studies with slight modifications [11]. In brief, lentivirus expressing either ZFX-specific siRNA or scrambled siRNA was added into human tongue squamous cell carcinoma cell line Tca-8113. After incubation for another 4 days, cells were resuspended and seeded in 6-cm dishes. Cells were collected and fixed with ice-cold $70 \%$ alcohol after reaching approximately $80 \%$ coverage. $40 \times \mathrm{PI}$ stock $(2 \mathrm{mg} / \mathrm{ml}), 100 \times$ RNase stock $(10 \mathrm{mg} / \mathrm{ml})$, and $1 \times$ PBS buffer at a dilution of 25:10:1000 were used for subsequent cell staining. Then, flow cytometry machine FACS Calibur (Becton-Dickinson, USA) was used to perform cell cycle analysis. At least $1 \times 10^{6}$ cells per sample were used for cell cycle analysis, and experiments were replicated for three times independently.

\section{Western blot}

In brief, Tca- 8113 cells were washed with TBST buffer and then treated with lysis buffer. Appropriate protein lysates were mixed with $2 \times$ loading buffer, separated on 10-12\% SDSPAGE and transferred to PVDF membranes (Amersham), which was further blocked with $5 \%$ dissolved milk buffer for $2 \mathrm{~h}$ at room temperature and incubated overnight with specific primary antibodies including anti-cleaved caspase 3 (Cell Signaling \#9664), anti-cleaved PARP (Cell Signaling \#5625), and anti-GAPDH. After washing three times with TBST buffer, corresponding secondary antibodies coupled to HRP from Santa Cruz were added and ECL-Plus kit (Amersham Biosciences) was used for signal detection. 


\section{Results}

Increased ZFX expression in tumors of tongue SCC patients

Initially, tumor tissues and adjacent tumor tissues collected from tongue SCC patients were examined and confirmed using hematoxylin and eosin $(\mathrm{H} \& \mathrm{E})$ staining (representative images, Fig. 1a-b). Then, ZFX expression status was examined using immunohistochemistry (IHC) staining in tumor and adjacent normal tissues (representative images, Fig. 1a-b). ZFX expression were further quantified depending on the optical density of IHC signals and compared between tumors and adjacent normal tissues. Results showed that ZFX expression in tumor tissues was significantly higher than that in adjacent normal tissues (Fig. 2).

ZFX expression was suppressed using lentiviral-mediated siRNA strategy in Tca-8113 cell lines

As described above, ZFX expression in human tongue SCC samples was significantly higher than that in normal adjacent tissues, which implicated that ZFX might be a pathological factor involved in human tongue SCC development. Tca-8113 cell line from human tongue squamous cell carcinoma was selected for ZFX functional analysis in human tongue SCC development in vitro. And, lentiviral-mediated small interfering RNA (siRNA) strategy was employed to inhibit ZFX

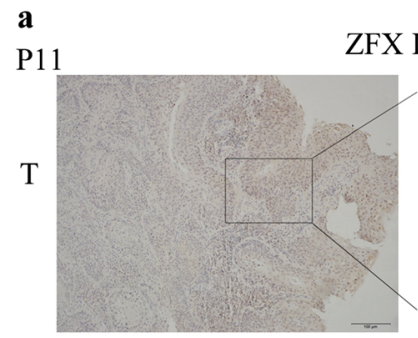

ZFX IHC
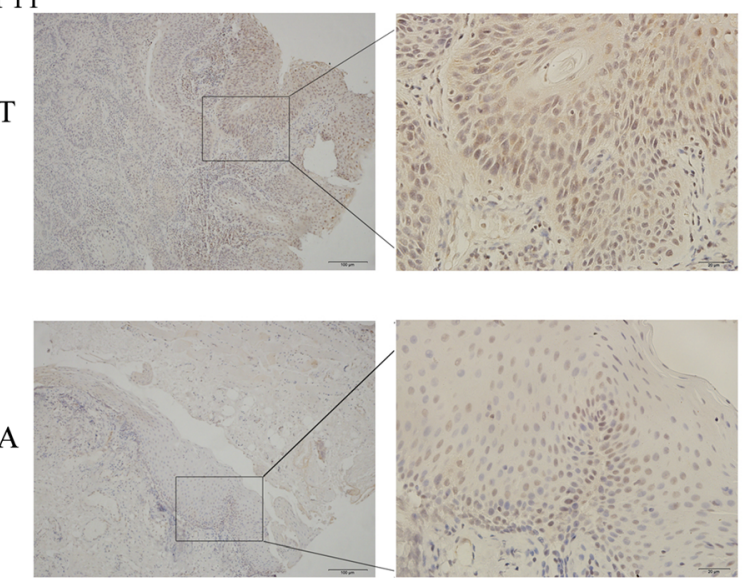

$100 \times$

b
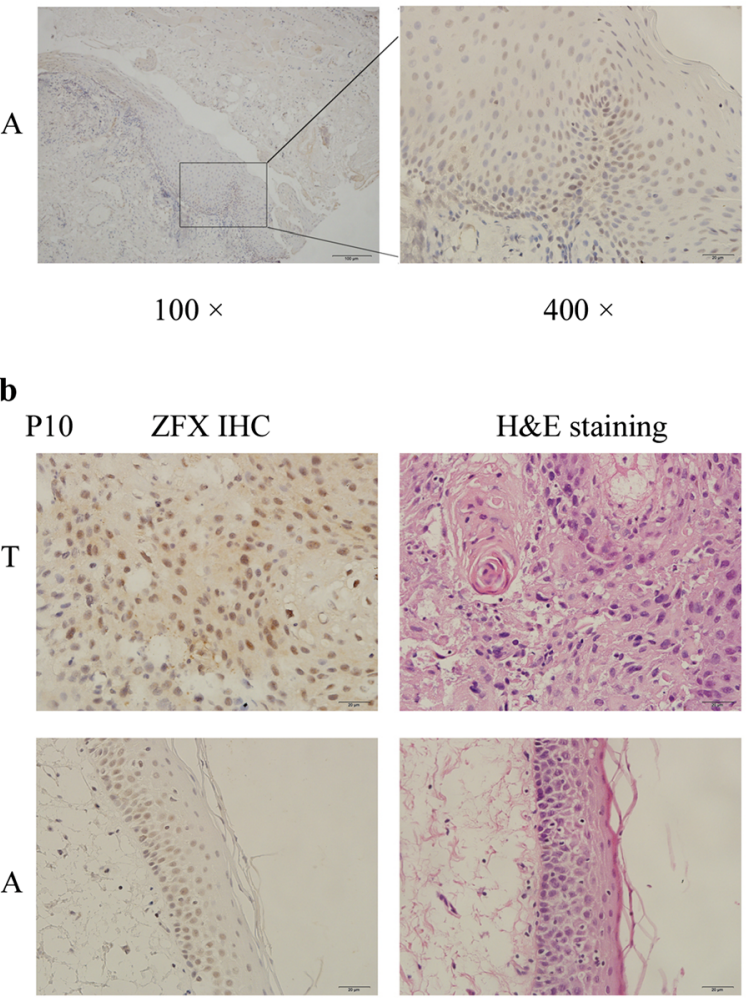

$400 \times$
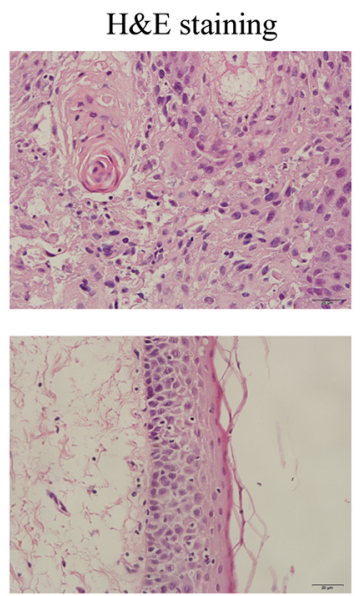

$400 \times$

Fig. 1 Expression of ZFX proteins in tumors and adjacent normal tissues from tongue SCC patients using IHC immunohistochemistry (IHC) and H\&E staining. $T$ tumor tissues, $A$ adjacent normal tissues, $P 11$ patient 11 , $P 10$ patient $10, P 15$ patient 15 . a Sample micrographs of ZFX IHC and $\mathrm{H} \& \mathrm{E}$ staining in tongue SCC tumor tissues and adjacent normal tissues
H\&E staining
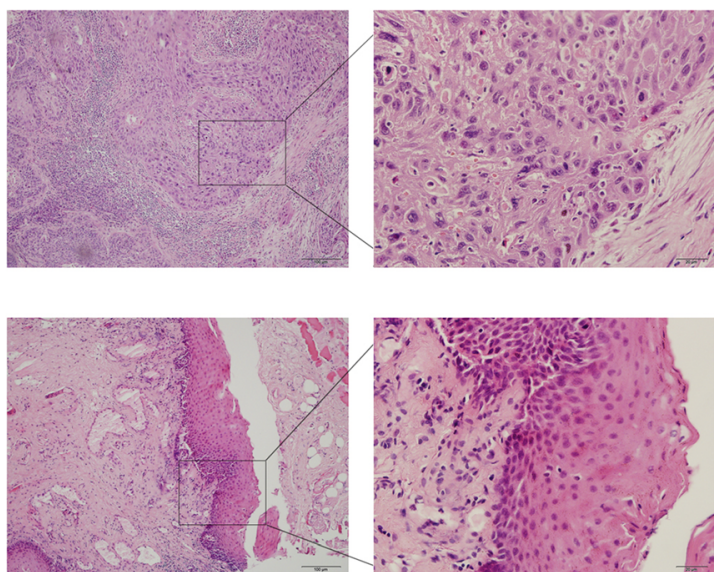

$100 \times$

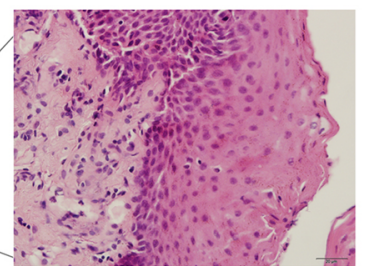

$400 \times$

P15 ZFX IHC
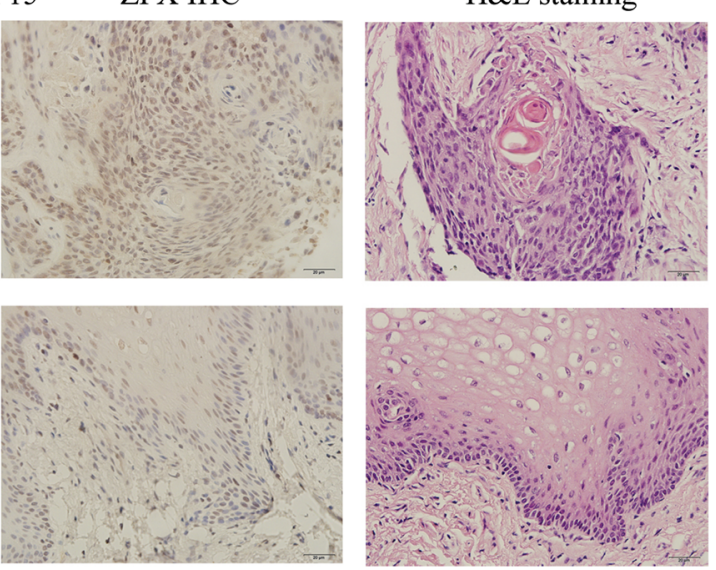

$400 \times$

from patient 11 (left, 100×, right, 400×). b Sample micrographs of ZFX staining and H\&E staining in tongue SCC tumor tissues and adjacent normal tissues from patient 10 and patient 15 (left, patient 10, right, patient 15 ; magnification, $400 \times$ ) 


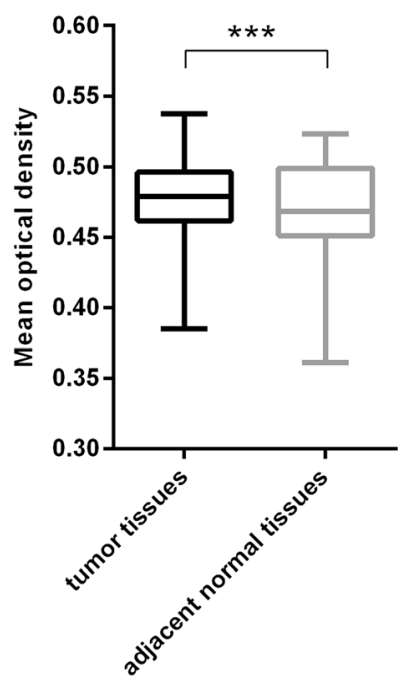

Fig. 2 Expression quantification of ZFX proteins in tumor and adjacent normal tissues. Quantification of ZFX expression in tumors and tumoradjacent normal tissues depending on optical density of ZFX positive signals in IHC slices

expression in Tca-8113 cells. Lentivirus expressing either scrsiRNA or ZFX-siRNA was generated and added into Tca8113 cells. GFP expression could be observed in more than $95 \%$ of cells $48 \mathrm{~h}$ after lentivirus tranfection (Fig. 3a). After $48 \mathrm{~h}$ incubation, total RNAs were extracted from cells infected with lentivirus expressing scr-siRNA or ZFX-siRNA and ZFX expression status at mRNA level was determined using real-time quantitative PCR. It was shown that ZFX expression in cells infected with ZFX-siRNA was downregulated by $73 \%$ as compared to cells infected with scr-siRNA (Fig. 3b). Our results provided strong evidence showing that lentiviral-mediated siRNA strategy could inhibit ZFX expression efficiently in Tca- 8113 cells, which provided a reliable method for ZFX functional analysis in subsequent experiments.
ZFX knockdown inhibited cell proliferation inTca-8113 cells

Sustained proliferation ability is the most fundamental hallmark of cancer cells. So, here, Tca- 8113 cells were treated with lentivirus expressing ZFX- or scr-siRNA, and BrdU incorporation assay was employed to investigate the impact of ZFX knockdown on cell proliferation ability. BrdU incorporation ratio was determined after $24 \mathrm{~h}$ of cell seeding, and it was shown that initial proliferation ability of Tca- 8113 cells with different treatment was comparable (Fig. 4a, day 1). However, after 4 days of lentivirus infection, ZFX-specific siRNA resulted in remarkably impaired cell proliferation with about $24 \%$ reduction of BrdU incorporation ratio (Fig. 4a, day 4). Cell proliferation status was further analyzed with MTT assay for continuous 5 days. At the first day, cell proliferation was comparable in Tca-8113 cells treated with either scrsiRNA or ZFX-siRNA. However, in the following 4 days, cell proliferation was significantly impaired in cells treated with ZFX-siRNA as compared to cells treated with scr-siRNA (Fig. 4b). Our results revealed that when ZFX was inhibited, Tca-8113 cell proliferation was suppressed significantly.

Impairment of colony formation ability in Tca- 8113 cells by ZFX knockdown

Clonogenic ability is critical for tumor outgrowth and expansion and could be determined using colony formation assay, which examines the ability of a single cell to form a colony containing at least 50 cells. And here, it was shown that smaller clones containing sparse and fewer cells were formed by Tca- 8113 cells targeted by ZFX-siRNA as compared to clones formed by Tca- 8113 cells targeted by scr-siRNAs (Fig. 4c, left panel). Furthermore, fluorescence in clones formed by ZFXsiRNA-treated cells was significantly weaker than that in a

Scr-siRNA
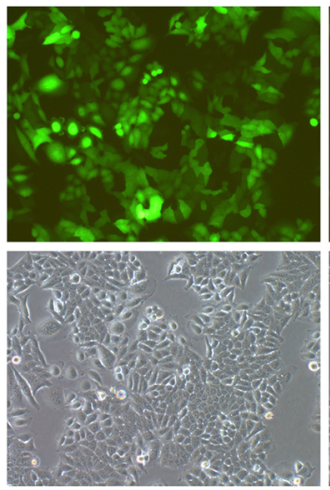

ZFX-siRNA
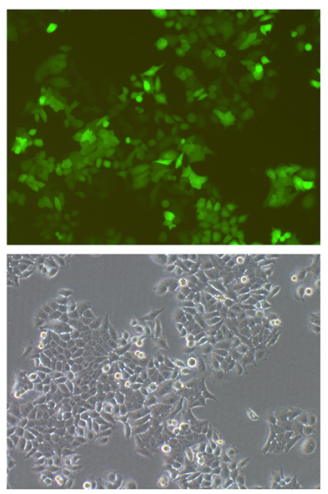

b

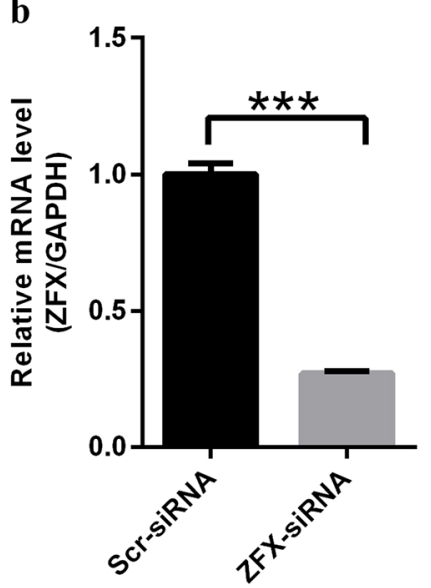

Fig. 3 ZFX knockdown by lentiviral-based siRNA strategy. a Representative pictures of Tca- 8113 cells infected with lentivirus expressing either scr-siRNA or ZFX-siRNA for $48 \mathrm{~h}$. b ZFX expression at mRNA level in cells infected with lentivirus expressing either scr-siRNA or ZFX-siRNA was analyzed using real-time quantitative PCR method. GAPDH was used as internal control, and data shown here is the mean \pm S.D. of three independent experiments $(* * * p<0.001)$ 
a

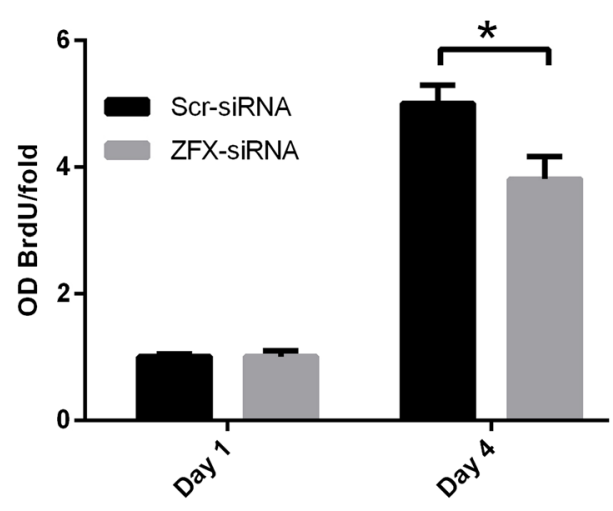

c Scr-siRNA

ZFX-siRNA
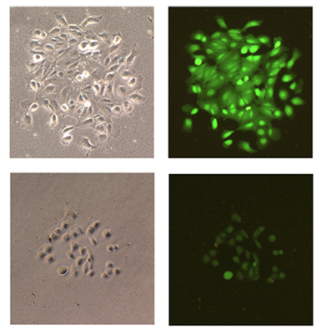

d

Scr-siRNA
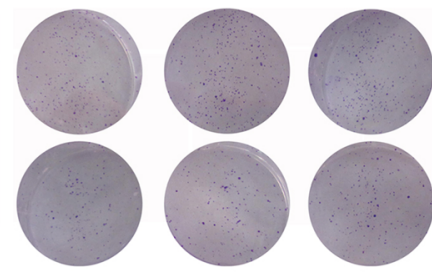

ZFX-siRNA

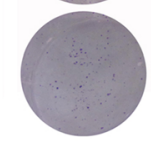

Fig. 4 Impairment of cell proliferation and colony formation ability in Tca-8113 cells infected with lentivirus expressing ZFX-siRNA. a BrdU incorporation assay was used to analyze the proliferation of Tca-8113 cells infected with lentivirus expressing either scr-siRNA or ZFXsiRNA. The BrdU incorporation ratio is represented as fold changes of absorbance at $450 \mathrm{~nm}\left(\mathrm{OD}_{\mathrm{BrdU}} /\right.$ fold $)$. Day 1 means the $\mathrm{BrdU}$ incorporation ratio in cells $24 \mathrm{~h}$ after lentivirus infection, and day 4 means the BrdU incorporation ratio 4 days after lentivirus infection. Data here is the mean \pm S.D. of three independent experiments $\left({ }^{*} p<0.05\right)$. b Cell proliferation of Tca- 8113 cells infected with lentivirus expressing either scr-siRNA or ZFX-siRNA was further

clones formed by scr-siRNA-treated cells (Fig. 4c, right panel). And, colony formation assay showed that an average of 224 clones formed in scr-siRNA-treated cells while the clone number was reduced to 185 in ZFX-siRNA-treated cells (Fig. 4d-e). These results revealed that ZFX knockdown inhibited clone formation in Tca- 8113 cells, which indicated that ZFX is essential for clonogenic ability of human tongue squamous cell carcinoma cells.

\section{Cell cycle disruption by ZFX knockdown}

Impairment of cell proliferation by ZFX knockdown promoted us to examine the relationship between ZFX and cell cycle b
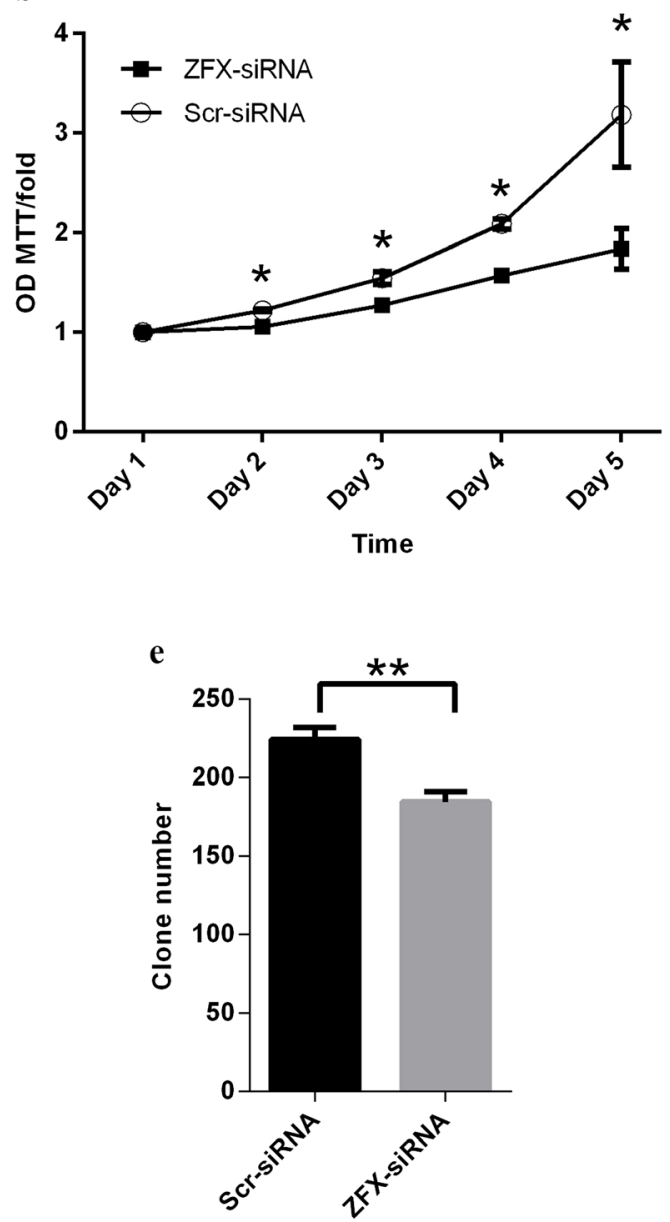

determined by MTT assay for continuous 5 days. Data shown here is the mean \pm S.D. from three independent experiments $\left({ }^{*} p<0.05\right)$. c Representative pictures of clones formed by Tca- 8113 cells infected with lentivirus expressing either scr-siRNA or ZFX-siRNA. Differential interference contrast (DIC, left panel) and GFP (right panel) pictures were shown. d Representative pictures of colony formation assay in 6well plate for Tca- 8113 cells infected by lentivirus expressing either scrsiRNA or ZFX-siRNA. e Quantification of clone number in Tca-8113 cells infected by lentivirus expressing either scr-siRNA or ZFX-siRNA. Data shown here is the mean \pm S.D. of clone numbers from three independent experiments $(* * p<0.01)$

progression in Tca-8113 cells. Cells were treated with lentivirus expressing either scr-siRNA or ZFX-siRNA, and cell cycle was analyzed with flow cytometry (Fig. 5a). Results showed that significant cell cycle arrest at the G2/M phase was induced by ZFX knockdown, as the percentage of G2/ M phase cells increased from 7.59 to $12.27 \%$ with ZFXsiRNA treatment. In addition, cell percentage at $\mathrm{S}$ phage was significantly decreased from 40.85 to $35.08 \%$, but cell percentage at G1 phase changed little (Fig. 5b). Taken together, these data showed that cell cycle progression was disrupted by ZFX knockdown and indicated that ZFX knockdown-induced cell proliferation impairment might be partially attributed to cell cycle arrest. 
Fig. 5 Cell cycle disruption in Tca-8113 cells infected with lentivirus expressing ZFXspecific siRNA. a Cell cycle analysis by flow cytometry and representative images were shown. b Cell cycle distribution in Tca-8113 cells infected with lentivirus expressing scr-siRNA or ZFX-siRNA. Data shown here is the mean \pm S.D. of cell percentage from three independent experiments $\left({ }^{* *} p<0.01,{ }^{* * *} p<0.001\right)$
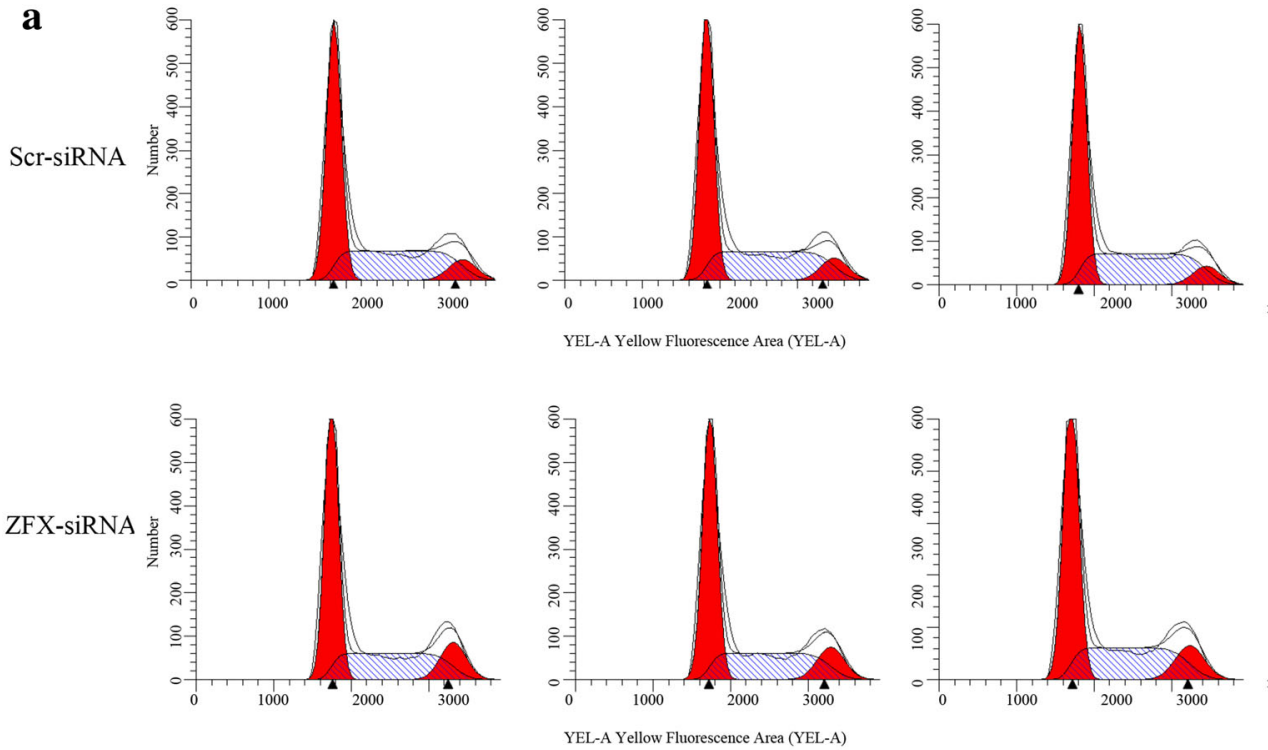

b

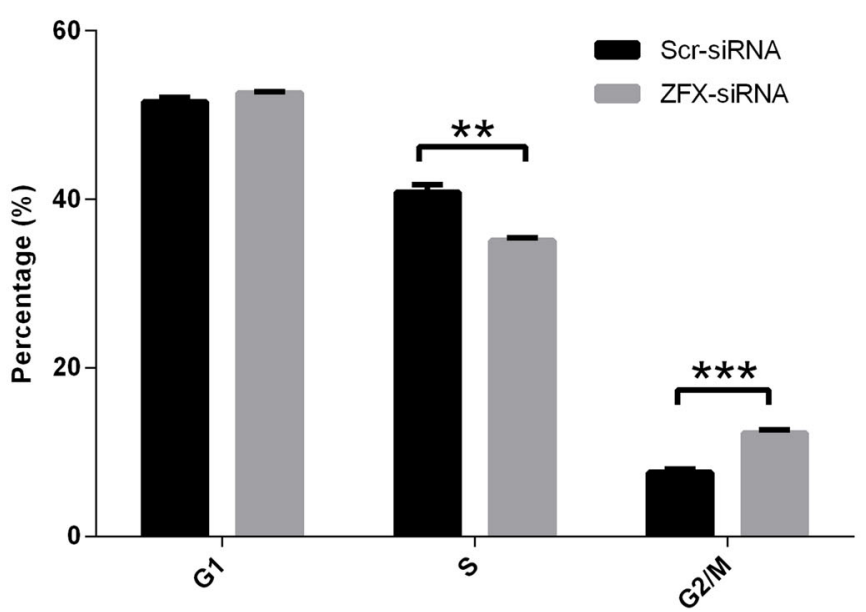

\section{Reduction of ZFX induced apoptosis in Tca- 8113 cells}

In addition to uncontrolled proliferation, apoptosis evasion is another fundamental trait of cancer cells. To examine the impact of ZFX knockdown on cell apoptosis, Tca-8113 cells were infected with lentivirus expressing ZFX-specific or scrambled siRNA. After 4 days of lentivirus infection, the apoptosis of cells was determined with annexin V-APC assay and analyzed by flow cytometry (Fig. 6a). Apoptosis was observed in $2.76 \%$ of Tca- 8113 cells infected with scrambled siRNA and increased to $3.5 \%$ in cells infected with ZFXspecific siRNA (Fig. 6b). Furthermore, Western blot analysis was performed to determine whether any apoptosisassociated genes were involved in the process of ZFXinduced cell apoptosis in Tca-8113 cells. It was shown that ZFX knockdown led to the upregulation of cleaved caspase 3 and PARP proteins (Fig. 6c), which were both critical for the induction of cell apoptosis. Taken together, these data suggests that ZFX plays an antiapoptotic role in Tca-8113 cells.

\section{Discussion}

ZFX protein is a $\mathrm{C} 2 \mathrm{H} 2-\mathrm{ZF}$ protein identified as a transcriptional factor belonging to ZFY protein family. Its involvement in cancer development has been explored extensively. For example, ZFX overexpression has been observed in prostate cancer [12], gastric cancer [13-15], human gliomas [16, 17], non-small-cell lung cancer (NSCLC) [18], breast cancer [19], and so on. Studies from our lab also showed that ZFX is involved in human laryngeal squamous cell carcinoma (LSCC), a subgroup of cancer of HNSCC, and ZFX knockdown impaired the cell proliferation and induced apoptosis in LSCC Hep-2 cells [7]. Here, we unraveled that ZFX expression increased significantly in tongue SCC tumors, another 
Fig. 6 Apoptosis induction in Tca-8113 cells infected with lentivirus expressing ZFXspecific siRNA. a Cell apoptosis by annexin-V staining was analyzed with flow cytometry. b Cell apoptosis in Tca-8113 cells infected with lentivirus expressing scr-siRNA or ZFXsiRNA. Apoptosis was determined with annexin V-APC assay and analyzed using flow cytometry. Data shown here is the mean \pm S.D. of cell percentage in apoptosis from three independent experiments $(* * p<0.01)$. c Western blot analysis of apoptosis-associated genes in Tca-8113 cell lysates infected with lentivirus expressing scr-siRNA or ZFX-siRNA. GAPDH was used as internal control

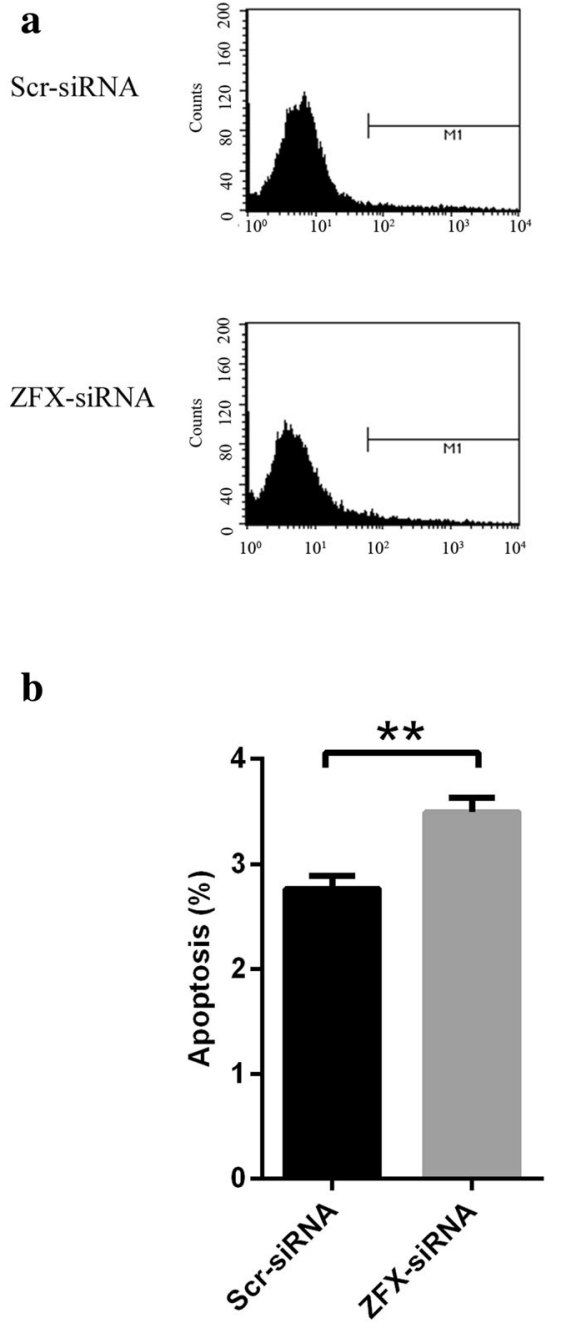

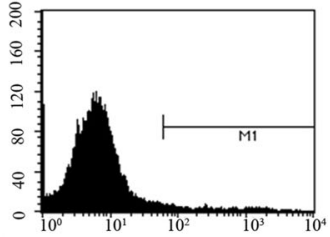

FL4-H

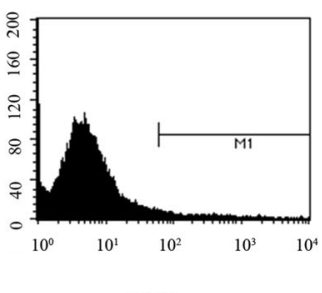

FL4-H

C
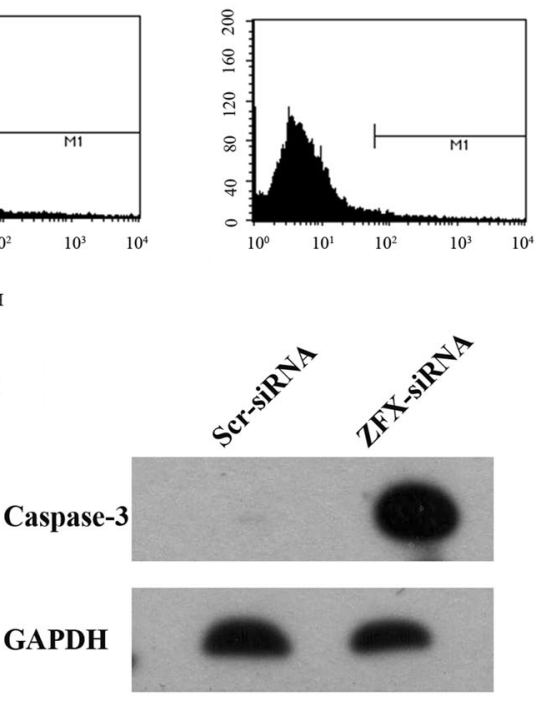

PARP

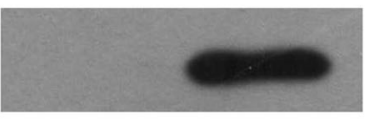

GAPDH

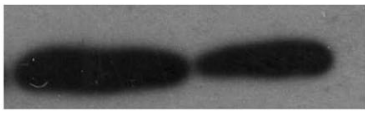

type of cancer of HNSCC, as compared to tumor-adjacent normal tissues. So, these results suggest that ZFX upregulation in tumors is quite common and ZFX as a potential biomarker for cancer diagnosis should be investigated in the future.

Tongue SCC accounts for about $90 \%$ of HNSCC, which ranks among the top ten cancers worldwide. However, disrupted molecular factors underlying tongue SCC initiation and progression remain poorly understood, which hindered the development of efficient strategies for tongue SCC prevention, diagnosis, prognosis, and treatment. Here, we quantified ZFX expression in tissue samples from tongue SCC patients by OD measurement rather than by pathologists, as OD measurement will provide objective quantifications about ZFX expression. And, our discovery of ZFX overexpression in tongue SCC samples expanded our understanding about molecular mechanisms of tongue SCC. Furthermore, in vitro studies in Tca- 8113 cells revealed that ZFX inhibition by lentivirus-based siRNA strategy delayed cell proliferation, impaired colony formation ability, and lead to cell cycle arrest while promoted cell apoptosis, suggesting its essential role for tumor cell growth and survival. However, the underlying mechanisms about colony formation ability impairment by ZFX knockdown remain elusive, though it was shown in this study that ZFX expression is essential for colony formation. In-depth analysis of ZFX-associated molecular pathways will provide more valuable insights in tongue SCC and HNSCC development.

Indeed, it has been reported that ZFX is the target gene of miR-144, and in consistent with the oncogenic functions of ZFX, miR-144 expression was diminished significantly in gastric cancer and non-small-cell lung cancer [14, 18], suggesting that disrupted miR-144-ZFX axis may be underlying the development of variant tumors. Studies also showed that miR-144 overexpression could inhibit the tumor growth and induce cell apoptosis in NSCLC which is in accordance with the effect of ZFX knockdown. In addition to the upstream effector miR-144, downstream effectors of ZFX were also 
investigated in other studies. It has been reported that ZFX knockdown in diverse tumor types including gliomas [17], NSCLC [20], LSCC [7], breast cancers [19], and gastric cancer [13] all led to hypophosphorylation of Akt, extracellular signal-regulated kinase 1/2 (ERK1/2), or MEK1/2 except for ERK2 hyperphosphorylation in breast cancer cells [19]. These results indicated that ZFX was critical for the activation of Akt and MAPK signaling pathways, which were two important pathways for cell survival. What's more, ZFX knockdown resulted in the downregulation of anti-apoptotic factor such as bcl-2 and upregulation of apoptotic factors including bax, caspase1, 3, 9 in diverse cancers [7, 12, 13, 17, 19, 20]. In our study, we also confirmed that two apoptosis-related proteins cleaved caspase 3 and PARP were both upregulated in Tca8113 cells treated with ZFX-specific siRNA. These results were all in consistent with the phenomenon that ZFX knockdown induces cell apoptosis in Tca-8113 cells and provided valuable insights for the underlying mechanisms of ZFXmediated cell apoptosis. Furthermore, it has been revealed that cell cyle factors such as cyclin D1 and cyclin B1 was downregulated by ZFX knockdown in multiple cancer types [17, $19,20]$, which might account for the cell cycle disruption in Tca-8113 cells treated with ZFX-siRNA. Indeed, ZFX knockdown induced G1 phase cell cycle arrest in Tca- 8113 cells, which is in consistent with previous cell cycle results in PC-3 cells [12], SGC7901 cells [13], H1299 cells [20], MDA-MB231 cells [19], U87, and U373 cells [21], and in contrast to results in MGC803 cells [13] and Hep-2 cells [7], in which S phase cell cycle arrest was induced by ZFX knockdown. It has been demonstrated that cyclin D1 and cyclin E1 were essential for $\mathrm{G} 1 / \mathrm{S}$ phase transition $[22,23]$ while cyclin $\mathrm{A} 2$ was involved in S-phase process [24], so it is quite likely that cyclin E1 or cyclin D1 function was disrupted in Tca- 8113 cells by ZFX knockdown, which has been confirmed in SGC7901 cells [13], U87, and U373 cells [17] treated with ZFX-siRNA. It should be noticed that though ZFX functions have been investigated in multiple cancers, very little research focused on the roles of ZFX in HNSCC or tongue squamous cell carcinoma. So, our future studies will focus on the upstream/ downstream pathways of ZFX in vitro and in vivo to provide more insights into the underlying mechanisms about HNSCC or tongue squamous cell carcinoma. A thorough and comprehensive analysis about ZFX in different cancers are also needed, and such analysis will help to clarify the distinct and overlapping roles of ZFX in different cancers, paving the way for the development of efficient clinical applications targeting ZFX.

Acknowledgment The paper was supported by Beijing City, the hospital authority clinical technology innovation project (grant no. XMLX201311); the National Natural Science Foundation of China(NSFC) (Grant No. 81272267) and "The priming scientific research foundation for the senior esearcher in Beijing Tongren Hospital, Capital Medical University".

\section{Conflicts of interest None}

Open Access This article is distributed under the terms of the Creative Commons Attribution License which permits any use, distribution, and reproduction in any medium, provided the original author(s) and the source are credited.

\section{References}

1. Warnakulasuriya S. Global epidemiology of oral and oropharyngeal cancer. Oral Oncol. 2009;45(4-5):309-16. doi:10.1016/j. oraloncology.2008.06.002.

2. Ganly I, Patel S, Shah J. Early stage squamous cell cancer of the oral tongue - clinicopathologic features affecting outcome. Cancer. 2012;118(1):101-11. doi:10.1002/cncr.26229.

3. Chaturvedi AK, Anderson WF, Lortet-Tieulent J, Curado MP, Ferlay $\mathrm{J}$, Franceschi S, et al. Worldwide trends in incidence rates for oral cavity and oropharyngeal cancers. J Clin Oncol: Off J Am Soc Clin Oncol. 2013;31(36):4550-9. doi:10.1200/JCO.2013.50.3870.

4. Ladomery M, Dellaire G. Multifunctional zinc finger proteins in development and disease. Ann Hum Genet. 2002;66(Pt 5-6):33142. doi:10.1017/S0003480002001215.

5. Galan-Caridad JM, Harel S, Arenzana TL, Hou ZE, Doetsch FK, Mirny LA, et al. Zfx controls the self-renewal of embryonic and hematopoietic stem cells. Cell. 2007;129(2):345-57. doi:10.1016/j. cell.2007.03.014.

6. Harel S, Tu EY, Weisberg S, Esquilin M, Chambers SM, Liu B, et al. ZFX controls the self-renewal of human embryonic stem cells. PLoS ONE. 2012;7(8):e42302. doi:10.1371/journal.pone.0042302.

7. Fang J, Yu Z, Lian M, Ma H, Tai J, Zhang L, et al. Knockdown of zinc finger protein, X-linked (ZFX) inhibits cell proliferation and induces apoptosis in human laryngeal squamous cell carcinoma. Mol Cell Biochem. 2012;360(1-2):301-7. doi:10.1007/s11010011-1069-x.

8. Weisberg SP, Smith-Raska MR, Esquilin JM, Zhang J, Arenzana TL, Lau CM, et al. ZFX controls propagation and prevents differentiation of acute T-lymphoblastic and myeloid leukemia. Cell Rep. 2014;6(3): 528-40. doi:10.1016/j.celrep.2014.01.007.

9. Pena C, Garcia JM, Larriba MJ, Barderas R, Gomez I, Herrera M, et al. SNAI1 expression in colon cancer related with CDH1 and VDR downregulation in normal adjacent tissue. Oncogene. 2009;28(49): 4375-85. doi:10.1038/onc.2009.285.

10. Wang L, Zhou GB, Liu P, Song JH, Liang Y, Yan XJ, et al. Dissection of mechanisms of Chinese medicinal formula Realgar-Indigo naturalis as an effective treatment for promyelocytic leukemia. Proc Natl Acad Sci U S A. 2008;105(12):4826-31. doi:10.1073/pnas. 0712365105.

11. Sasaki K, Tsuno NH, Sunami E, Tsurita G, Kawai K, Okaji Y et al. Chloroquine potentiates the anti-cancer effect of 5-fluorouracil on colon cancer cells. BMC cancer. 2010;10. doi:10.1186/1471-240710-370.

12. Jiang H, Zhang L, Liu J, Chen Z, Na R, Ding G, et al. Knockdown of zinc finger protein $\mathrm{X}$-linked inhibits prostate cancer cell proliferation and induces apoptosis by activating caspase- 3 and caspase- 9 . Cancer Gene Ther. 2012;19(10):684-9. doi:10.1038/cgt.2012.53.

13. Wu S, Lao XY, Sun TT, Ren LL, Kong X, Wang JL, et al. Knockdown of ZFX inhibits gastric cancer cell growth in vitro and in vivo via downregulating the ERK-MAPK pathway. Cancer Lett. 2013;337(2):293-300. doi:10.1016/j.canlet.2013.04.003.

14. Akiyoshi S, Fukagawa T, Ueo H, Ishibashi M, Takahashi Y, Fabbri $\mathrm{M}$, et al. Clinical significance of miR-144-ZFX axis in disseminated tumour cells in bone marrow in gastric cancer cases. Br J Cancer. 2012;107(8):1345-53. doi:10.1038/bjc.2012.326. 
15. Nikpour P, Emadi-Baygi M, Mohammad-Hashem F, Maracy MR, Haghjooy-Javanmard S. Differential expression of ZFX gene in gastric cancer. J Biosci. 2012;37(1):85-90. doi:10.1007/s12038-0119174-2.

16. Zhou YX, Su ZP, Huang YL, Sun T, Chen SS, Wu TF et al. The Zfx gene is expressed in human gliomas and is important in the proliferation and apoptosis of the human malignant glioma cell line U251. J Exp Clin Canc Res. 2011;30. doi:10.1186/1756-9966-30-114.

17. Zhu ZC, Li K, Xu DF, Liu YJ, Tang HL, Xie Q, et al. ZFX regulates glioma cell proliferation and survival in vitro and in vivo. J NeuroOncol. 2013;112(1):17-25. doi:10.1007/s11060-012-1032-Z.

18. Zha WJ, Cao L, Shen Y, Huang M. Roles of Mir-144-ZFX Pathway in growth regulation of non-small-cell lung cancer. PloS one. 2013;8(9). doi:10.1371/journal.pone.0074175.

19. Yang HJ, Lu Y, Zheng YB, Yu XF, Xia XH, He XM, et al. shRNAmediated silencing of ZFX attenuated the proliferation of breast cancer cells. Cancer Chemother Pharmacol. 2014;73(3):569-76. doi:10. 1007/s00280-014-2379-y.
20. Li K, Zhu ZC, Liu YJ, Liu JW, Wang HT, Xiong ZQ, et al. ZFX knockdown inhibits growth and migration of non-small cell lung carcinoma cell line H1299. Int J Clin Exp Pathol. 2013;6(11):2460-7.

21. Zhu ZC, Li K, Xu DF, Liu YJ, Tang HL, Xie Q, et al. ZFX regulates glioma cell proliferation and survival in vitro and in vivo (vol 112, pg 17, 2013). J Neuro-Oncol. 2013;113(3):533. doi:10.1007/s11060013-1174-7.

22. Ye X, Nalepa G, Welcker M, Kessler BM, Spooner E, Qin J, et al. Recognition of phosphodegron motifs in human cyclin E by the SCF (Fbw7) ubiquitin ligase. J Biol Chem. 2004;279(48):50110-9. doi: 10.1074/jbc.M409226200.

23. Lukas J, Bartkova J, Bartek J. Convergence of mitogenic signalling cascades from diverse classes of receptors at the cyclin D-cyclindependent kinase-pRb-controlled G1 checkpoint. Mol Cell Biol. 1996;16(12):6917-25.

24. Pagano M, Pepperkok R, Verde F, Ansorge W, Draetta G. Cyclin-a is required at 2 points in the human cell cycle. Embo J. 1992;11(3):96171. 